\title{
Case Report: A Rare Association of Diffuse Thyroid Lipomatosis with Amyloid Deposition
}

\author{
Daniela Rodrigues Cavaco ${ }^{a} \quad$ Ana Alves Rafael ${ }^{b} \quad$ Rafael Cabrera $^{c}$ Helena Vilar ${ }^{a}$ \\ Valeriano Leite ${ }^{a, d}$ \\ aDepartment of Endocrinology, Instituto Português de Oncologia de Lisboa Francisco Gentil, Lisbon, Portugal; \\ bDepartment of General Surgery, CUF Academic Center - Hospital CUF Sintra and Hospital CUF Cascais, Lisbon, \\ Portugal; 'Department of Pathology, Instituto Português de Oncologia de Lisboa Francisco Gentil, Lisbon, Portugal; \\ dNOVA Medical School/New University of Lisboa, Lisbon, Portugal
}

\section{Established Facts}

- Diffuse thyroid lipomatosis is a rare histopathological condition of unknown etiology, characterized by diffuse fatty infiltration of the thyroid.

- This condition can result in diffuse goiter with compressive symptoms.

\section{Novel Insights}

- This is one of the few reported cases of diffuse lipomatosis with coexisting deposition of amyloid in the thyroid gland.

\section{Keywords}

Diffuse thyroid lipomatosis · Amyloid goiter

\begin{abstract}
Diffuse thyroid lipomatosis is a rare histopathological condition of unknown etiology, characterized by diffuse fatty infiltration of the thyroid stroma, which can result in diffuse goiter with compressive symptoms. We report a case of a 46-year-old man with 1-year history of progressive goiter enlargement with compressive symptoms. Imaging studies re-
\end{abstract}

vealed multiple coalescent nodules. The patient underwent surgery, and the microscopic appearance revealed a diffuse infiltration of thyroid stroma by mature adipose tissue with associated amyloid deposition. A final diagnosis of diffuse lipomatosis of the thyroid gland was established. This patient represents one of the few reported cases of diffuse lipomatosis with coexisting deposition of amyloid protein of the thyroid gland and contributes to the better understanding of this extremely rare condition.

(C) 2021 European Thyroid Association. Published by S. Karger AG, Basel
Correspondence to:

Daniela Rodrigues Cavaco, daniela.rcavaco@gmail.com 


\section{Introduction}

Diffuse thyroid lipomatosis is a histopathological condition of unknown etiology characterized by extensive diffuse fatty infiltration of the thyroid stroma. In some rare cases, it has been associated with the deposition of amyloid in the thyroid gland $[1,2]$. This condition can lead to the enlargement of the gland and can result in compressive symptoms [1]. Extensive adipose tissue in the thyroid gland can be found in a broad spectrum of conditions, from malignant lesions (thyroid carcinomas) to benign conditions (such as Graves' disease) [2,3]. Diffuse thyroid lipomatosis (or thyrolipomatosis) and thyrolipoma (adenolipoma) are part of the benign spectrum of lesions of the thyroid gland. The diffuse form is even less common than thyrolipoma and is characterized by mature adipose tissue deposition without evidence of encapsulation [4]. On the other hand, amyloid goiter results from the deposition of insoluble proteins due to its abnormal folding process and also can lead to the deposition of adipose tissue within the thyroid gland $[5,6]$. A case of diffuse thyroid lipomatosis with coexistent deposition of amyloid protein is presented.

\section{Case Presentation}

We report the case of a 46-year-old man with a history of bronchiectasis with multiple episodes of viral and bacterial respiratory infections since he was 19 years old, chronic otitis media, pansinusitis, and infertility due to idiopathic hypogonadotropic hypogonadism. The patient referred a history of consanguinity (2 grandparents are brothers, and his parents are first-degree cousins). He has 2 children, both born after medically assisted reproduction, diagnosed with severe immunodeficiency with frequent respiratory and central nervous system infections. The patient presented with a 2 -year history of progressive weight loss $(<10 \%$ of total body weight) associated with fatigue to mild effort. Physical examination showed a lobulated diffuse thyroid enlargement and multiple inflammatory lymph nodules in the lateral compartments of the neck. Routine blood tests were normal, including cell blood count, renal function, proteinogram, beta- 2 microglobulin, immunoglobulins, and C-reactive protein. The sedimentation rate was 35 (normal range $0-22 \mathrm{~mm} / \mathrm{h}$ ).

Thyroid function tests were within normal range with free T4 $1.46 \mathrm{pg} / \mathrm{dL}$ (NR 0.90-1.70), free T3 $3.3 \mathrm{pg} / \mathrm{mL}$ (NR 2.0-4.5), and TSH $1.35 \mu \mathrm{UI} / \mathrm{mL}$ (NR 0.30-4.20). Serum anti-thyroperoxidase and anti-thyroglobulin antibodies as well as serum calcitonin levels were undetectable. Two months later, neck ultrasound (US) scan revealed thyroid enlargement with a right thyroid lobe of 65 $\times 32 \times 34 \mathrm{~mm}$ and a left lobe of $64 \times 31 \times 28 \mathrm{~mm}$ with iso-hypoechogenic and solid nodules with $18 \mathrm{~mm}$ and $15 \mathrm{~mm}$ in the right and left lobes, respectively, without cervical lymphadenopathy. FNA was performed in both nodules, which showed colloid goiter. The CT scan revealed a diffuse enlarged thyroid with areas of low-

Diffuse Thyroid Lipomatosis with

Amyloid Deposition

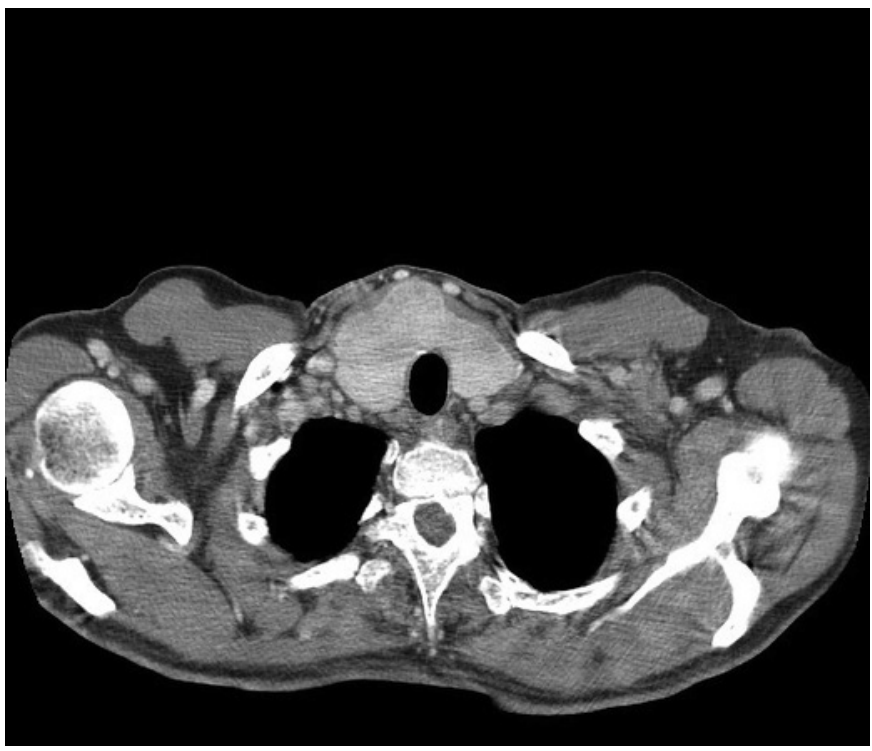

Fig. 1. CT scan showing diffuse thyroid enlargement with hypodense areas.

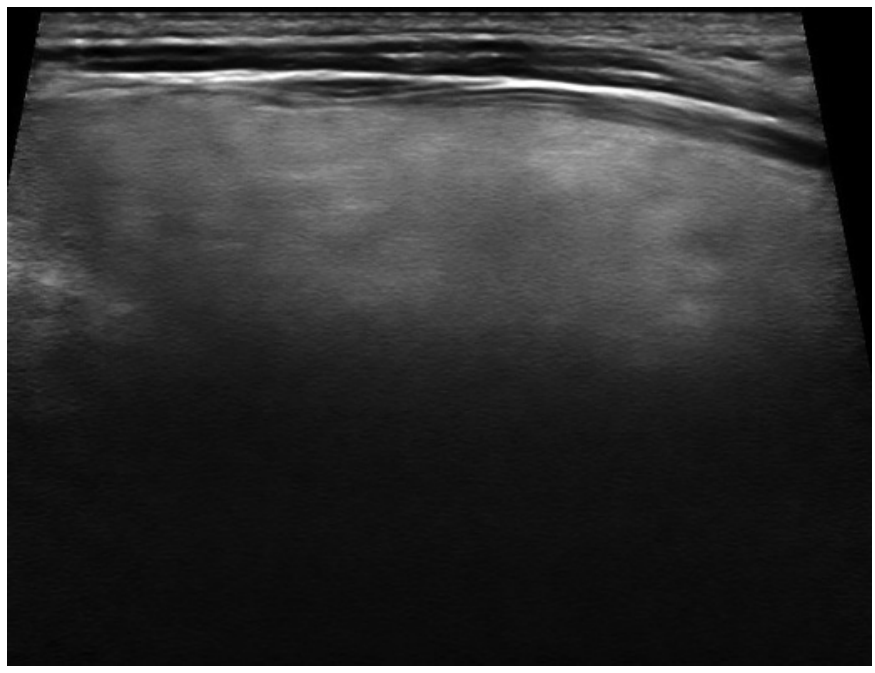

Fig. 2. Thyroid ultrasound: the thyroid left lobe showing diffuse hyperechogenicity.

er density than the remaining parenchyma (Fig. 1). The trachea was slightly deviated to the left side without compression. No cervical or mediastinal suspicious lymph nodes were detected. Twelve months later, the goiter enlarged, and he developed symptoms of dyspnea, dysphonia, and dysphagia. Neck US revealed coalescent iso-hyperechogenic nodules in the thyroid gland, the largest with $20 \mathrm{~mm}$ (Fig. 2), and FNA was repeated in this nodule with the diagnosis of a follicular lesion of undetermined significance, showing scarce cellularity, abundant colloid, macrophages, and some follicular cells with nuclear alterations (slight nuclear increase, clear chromatin, membrane irregularity, and grooves). 
Fig. 3. a Histologically, there was infiltration of thyroid stroma by mature adipose tissue (black arrow). b Interstitial amyloid deposition stained with Congo red (black arrow) showing apple-green birefringence under polarized light (yellow arrow).
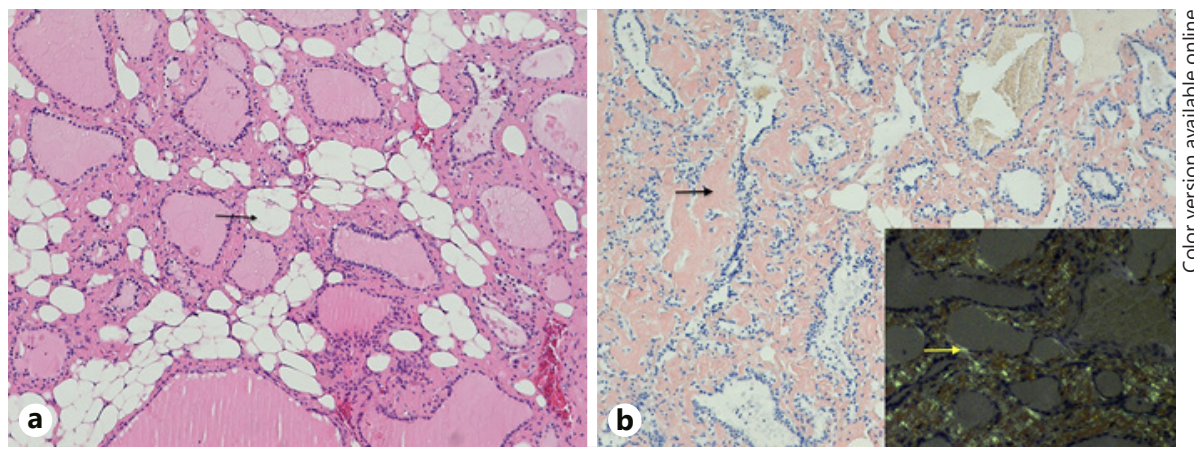

Table 1. Patient characteristics and imaging findings for the cases of diffuse thyroid lipomatosis and amyloid deposition reported in the English literature

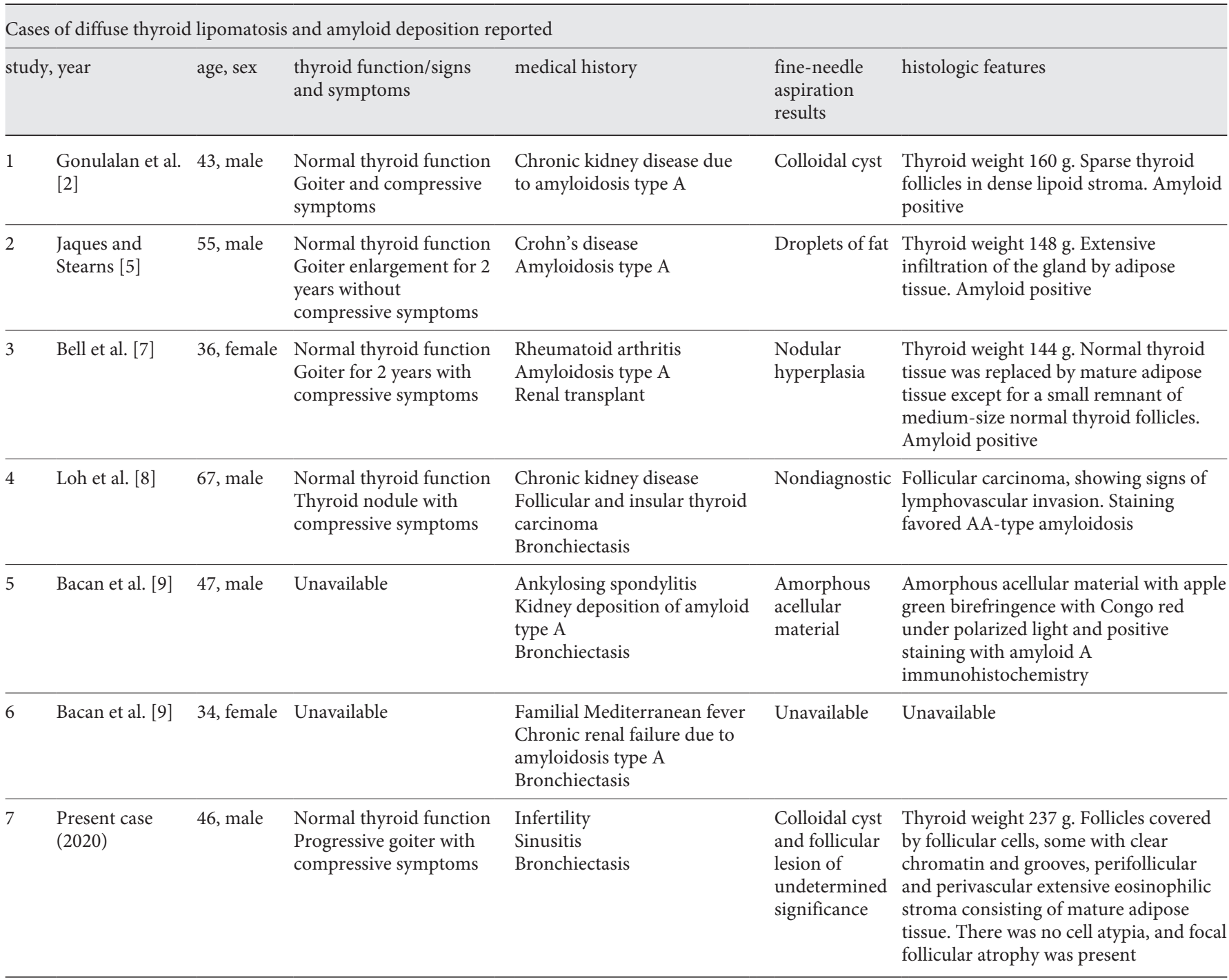


A total thyroidectomy was performed without surgical complications. The macroscopic appearance of the resected thyroid showed a bosselated, homogenous 237-g thyroid with yellow and hemorrhagic areas, without visible nodules. The left lobe measured $111 \times 40 \times 26 \mathrm{~mm}$ and the right lobe $105 \times 65 \times 43 \mathrm{~mm}$. Microscopic examination of multiple sections revealed normal follicular architecture, with normal appearing follicular cells. There was fatty infiltration of the perifollicular and perivascular stroma, with extracellular deposition of an amorphous eosinophilic material, consistent with amyloid. There was apple-green birefringence of Congo red-stained slides under polarized light, confirming the presence of amyloid protein (Fig. 3). A final diagnosis of diffuse lipomatosis of the thyroid gland with amyloid deposition was established. There was no evidence of systemic amyloidosis. Abdominal fat and bone marrow biopsies revealed no pathological alterations. ${ }^{18}$ FDG-PET scan presented a faint lung uptake probably related to the bronchiectasis. The electrocardiogram and echocardiogram showed no abnormalities. Alpha-1 antitrypsin deficiency, familial Mediterranean fever, inflammatory bowel disease, cystic fibrosis, and idiopathic pulmonary fibrosis were also ruled out. The patient remains asymptomatic under treatment with levothyroxine.

\section{Discussion}

This case represents one of few published cases of diffuse thyroid lipomatosis. With the keywords "diffuse thyroid lipomatosis," "thyrolipoma," and "thyrolipomatosis" in the PubMed, we could find only 39 case reports and only 6 also had concomitant amyloid deposition (Table 1). These patients usually presented with normal thyroid function and severe compressive symptoms $[2,3,7$, 8, 10]. Bakan et al. [9] and Loh et al. [8] described 3 patients with coexistent bronchiectasis as in our patient (Table 1) [11]. Amyloid deposition can also lead to massive goiters and be associated with variable amounts of adipose tissue within the thyroid gland; however, the majority of the cases of diffuse thyroid lipomatosis do not have significant amyloid content $[5,6]$.

The patient and his children have an unclear immunodeficiency disorder with severe and recurrent infections, which is still under investigation. Although it is consensual that amyloid deposition can occur as a reaction to systemic disease, such as a chronic inflammatory disease or a chronic infection, the pathophysiology underlying the association between amyloidosis and fat tissue deposition (especially in a massive way) remains to be elucidated. One possibility is capillary injury induced by amyloid, which leads to ischemia and subsequent metaplasia of stromal fibroblasts $[5,6,10]$. In addition, adipose tissue proliferation within the thyroid has been associated by some authors with disturbed embryogenesis $[3,4]$. To the

Diffuse Thyroid Lipomatosis with

Amyloid Deposition best of our knowledge, this case represents one of the few patients reported with diffuse thyroid lipomatosis with coexistent deposition of amyloid. Despite its rarity, thyroid lipomatosis should be considered in the differential diagnosis of a patient presenting with a goiter, and the clinician must be aware of the possibility of underlying systemic diseases.

\section{Acknowledgment}

The case report has been presented in the 42nd Annual Meeting of the European Thyroid Association that was held in Budapest, Hungary, in September 2019.

\section{Statement of Ethics}

The authors declare that the procedures were followed according to the regulations established by the Clinical Research and Ethics Committee and the Helsinki Declaration of the World Medical Association. The authors declare that the manuscript, complete or in parts, does not infringe any copyright and does not violate any privacy rights. The authors declare that no experiments were performed on humans or animals for this investigation. The authors state that the subject has given his written informed consent to publish his case, including publication of images.

\section{Conflict of Interest Statement}

The authors declared no potential conflicts of interest with respect to the research, authorship, and/or publication of this article.

\section{Funding Sources}

The authors received no financial support for the research, authorship, and/or publication of this article.

\section{Author Contributions}

We declare that all authors have contributed substantially to the manuscript, have approved the final version of the manuscript, and the manuscript is not under review or has been published, completely or partially, in any other journal.

\section{Data Availability Statement}

The authors confirm the data supporting the findings of this case report are available within the article. Raw data that support the findings of this case report are also available from the corresponding author, upon reasonable request. 


\section{References}

1 Arslan A, Alíç B, Uzunlar AK, Büyükbayram $\mathrm{H}$, Sarí I. Diffuse lipomatosis of thyroid gland. Auris Nasus Larynx. 1999 Jul;26(2):213-5.

2 Gonulalan G, Esen H, Erikoğlu M, Cakir M. Thyroid lipomatosis. Intern Med. 2012 Sep; 51(24):3383-5.

3 Hijazi DM, Addas FA, Alghanmi NM, Marzouki HZ, Merdad MA. An enlarged goiter presenting with a rare diffuse lipomatosis of the thyroid gland. Am J Case Rep. 2018 Jul;19: 808-11.

4 Ge Y, Luna MA, Cowan DF, Truong LD, Ayala AG. Thyrolipoma and thyrolipomatosis: 5 case reports and historical review of the literature. Ann Diagn Pathol. 2009;13(6):384-9.
5 Jacques TA, Stearns MP. Diffuse lipomatosis of the thyroid with amyloid deposition. J Laryngol Otol. 2013 Jan;127(4):426-8.

6 Cannizzaro MA, Lo Bianco S, Saliba W, D'Errico S, Pennetti Pennella F, Buttafuoco G, et al. A rare case of primary thyroid amyloidosis. Int J Surg Case Rep. 2018 Oct;53:17981.

7 Bell S, Sosa GA, Valle Jaen A, Picasso MF. Thyroid lipomatosis in a 36-year-old patient with rheumatoid arthritis and a kidney transplant. Endocrinol Diabetes Metab Case Rep. 2016;2016:160007.

8 Loh TL, Latis S, Ali RB, Patel H. Insular carcinoma arising on a background of follicular carcinoma, thyrolipomatosis and amyloid goitre. BMJ Case Rep. 2017;2017: bcr2017219747.
9 Bakan S, Kandemirli SG, Akbas S, Cingoz M, Ozcan Guzelbey B, Kantarci F, et al. Amyloid goiter: a diagnosis to consider in diffuse fatty infiltration of the thyroid. J Ultrasound Med. 2017 Aug;36(5):1045-9.

10 Schroder S, Bocker W. Lipomatous lesions of the thyroid gland: a review. Appl Pathol. 1985; 3(3): $140-9$.

11 López-Muñoz B, Greco Bermúdez L, MarínJiménez D, Sánchez de la Fuente MF, Capparelli AF, Mascarell Martínez I, et al. An unusual amyloid goiter in a 48-year-old woman with rheumatoid arthritis, secondary amyloidosis and renal failure. Case Rep Endocrinol. 2019 Dec;2019:4291486. 